\title{
COMPARATIVO DA RESISTÊNCIA DE UNIÃO IMEDIATA E APÓS ARMAZENAMENTO EM ÁGUA: REVISÃO DE LITERATURA
}

\section{COMPARATIVE STUDY OF IMMEDIATE BOND STRENGTH AND AFTER STORAGE IN WATER: A LITERATURE REVIEW}

\author{
Gislaine Cristine Martins* ; Vania Aparecida Oliveira Queiroz ${ }^{* *}$; \\ Marcio Rastelli*; Michele Bail ${ }^{*}$; João Carlos Gomes ${ }^{* * *}$; \\ Osnara Maria Mongruel Gomes ${ }^{* * * *}$
}

* Doutorandos do Curso de Pós Graduação em Odontologia, Universidade Estadual de Ponta Grossa, Ponta Grossa, Paraná, Brasil

** Professora Assistente. Disciplina de Dentística Operatória e Dentística Restauradora, Universidade Estadual de Ponta Grossa, Ponta Grossa, Paraná, Brasil

*** Professor Associado. Disciplina de Dentística Restauradora, Universidade Estadual de Ponta Grossa, Ponta Grossa, Paraná, Brasil

**** Professora Adjunta. Disciplina de Dentística Restauradora, Universidade Estadual de Ponta Grossa, Ponta Grossa, Paraná, Brasil

Recebido para publicação em 10/08/2010

Aceito para publicação em 18/12/2010

\section{RESUMO}

Os sistemas adesivos são classificados, atualmente, de acordo com a abordagem de união, ou seja, em convencionais ou autocondicionantes. Entretanto, a simplificação da técnica e do número de passos dos adesivos não pode afetar o seu desempenho em longo prazo, por isso, estudos que avaliam a resistência de união imediata e ao longo do tempo têm sido realizados com intuito de avaliar a interface adesiva. Para avaliação in vitro da longevidade tem sido utilizado o armazenamento em água ou em outras substâncias, uma vez que, pode promover um envelhecimento artificial. Desta forma, o objetivo deste trabalho foi revisar comparativamente a redução da resistência de união à dentina após armazenamento em água entre os sistemas adesivos convencionais e autocondicionantes. Após revisão de literatura, constatou-se que ao longo do tempo existe uma diminuição da resistência de união para a maioria dos adesivos, entretanto, essa diminuição é dependente do sistema adesivo utilizado. Palavras-chave: Adesivos dentinários. Resistência à tração. Longevidade.

\begin{abstract}
Adhesive systems are classified according to different adhesive bonding approaches; they can be either total or self-etch. However, the simplification of the technique and of the number of steps in the adhesive system should not affect its action over time. Therefore, studies that evaluate the immediate bond strength and throughout time have been carried out to evaluate the adhesive interface. To evaluate longevity, storage in water or in other substances has been used as they can promote artificial aging. Thus, the aim of this study was to review the literature about the reduction
\end{abstract}


of the dentin bond strength after storage in water of both total-etch and self-etch systems. After the literature review, it was observed that over time there is a decrease in the bond strength for most of the adhesives; however, the decrease varies according to the adhesive system used.

Keywords: Dentin-Bonding Agents. Tensile Strength. Longevity.

\section{Introdução}

Os sistemas adesivos são classificados, atualmente, de acordo com a abordagem de união, ou seja, em convencionais ou autocondicionantes e podem ser divididos de acordo com o número de passos, 3, 2 e 1 passo, respeitando os passos cardeais, ou seja, condicionamento ácido, primer e adesivo (bond). Os sistemas convencionais utilizam o condicionamento ácido como um passo separado, e podem ser encontrados em 3 e 2 passos (CARVALHO, 2004; VAN MEERBEEK， 2003).

Os fabricantes visando simplificar os procedimentos clínicos lançaram os sistemas adesivos que combinam o primer e o adesivo num único passo e, posteriormente os sistemas adesivos self-etching onde a hibridização ocorre simultaneamente com o condicionamento ácido, ou seja, em um único passo. A redução de passos é possível porque, nos sistemas adesivos autocondicionantes, a hibridização ocorre simultaneamente com o condicionamento ácido (BOUILLAGUET, 2001; ERNEST, 2004; VAN LANDUYT, 2006), pois apresentam um primer ácido e dispensam o passo do condicionamento ácido separadamente. Os sistemas autocondicionantes podem ser encontrados em 1 e 2 passos (CARVALHO, 2004; VAN MEERBEEK, 2003).

A redução do número de passos dos adesivos deve estar associada a um melhor desempenho em longo prazo e não apenas na simplificação da técnica. A integridade da união da interface adesiva tem implicações importantes no sucesso das restaurações de resina composta (DE MUNCK, 2003; 2005; CASTRO, 2004; AMARAL, 2007) e pode ser estudada avaliando a resistência de união, obtida pela adesão dos adesivos às resinas compostas e dos dois a estrutura dentária e, é de fundamental importância para avaliar a possível longevidade da restauração (DE MUNCK, 2003; CASTRO, 2004; GARCIA, 2002; SANO, 1999). Isso porque, atualmente o maior desafio é manter a união resina-dente estável por um tempo razoavelmente longo (DE MUNCK, 2003).

Estudos têm sido realizados com intuito de melhorar a adesão dos materiais restauradores à superfície dentária. A adesão dos adesivos às resinas compostas e dos dois a estrutura dentária é uma propriedade denominada resistência de união (DE MUNCK, 2003; CASTRO, 2004; SANO, 1999) que pode ser avaliada por ensaios mecânicos como, teste de cisalhamento (CASTRO, 2004), micro-cisalhamento, tração (GARCIA, 2002), ou microtração (SANO,1994; SCHREINER, 1998). O teste de microtração foi idealizado por Sano et al. em 1994 e permite analisar pequenas áreas de união, sendo possível avaliar vários espécimes de um mesmo substrato.

A resistência de união tem sido avaliada imediata (BOUILLAGUET, 2001, REIS, 2003; KNOBLOCH, 2007; OSÓRIO, 2008), após 6 meses (OSÓRIO, 2008; ARMSTRONG, 2003) ou mais de armazenamento (DE MUNCK, 2003). Estudos de longevidade são considerados um método ideal para validar a eficiência de restaurações e materiais adesivos (NIKAIDO, 2002; LOGUERCIO, 2005). O armazenamento pode promover um envelhecimento artificial e pode ser feito em água ou em outras substâncias (DE MUNCK, 2005; CASTRO, 2004; YAMAUTI, 2003).

Valores altos de resistência de união são necessários para garantir a retenção da restauração durante a contração de polimerização da resina composta, tensões mastigatórias, tensões térmicas $\mathrm{e}$, variações de $\mathrm{pH}$. Isso torna um desafio manter a integridade da união da interface adesiva, pois ela sofrem um processo degenerativo, pois, devido a fatores que podem causar degradação dessa interface de união ao longo do tempo (DE MUNCK, 2003, 2005). 
Por isso, o objetivo deste trabalho é revisar comparativamente a redução da resistência de união à dentina após armazenamento em água entre os sistemas adesivos convencionais e autocondicionantes.

\section{Revisão de Literatura}

Realizou-se busca nas bases de dados Medline. A busca para análise comparativa dos estudos "in vitro" da resistência de união imediata (ou até
24 horas) e após 6 meses de armazenamento em água foi limitada aos últimos 10 anos (2000 a 2010).

Foram selecionados os estudos "in vitro" que compararam a resistência de união à dentina (microtração) imediata e após 6 meses de armazenamento em água, dos sistemas adesivos convencionais (Tabela 1) e dos sistemas adesivos autocondicionantes (Tabela 2) que utilizaram dentes humanos. Cabe destacar que os dados coletados foram dos sistemas adesivos utilizados de acordo com as instruções dos respectivos fabricantes.

Tabela 1 - Médias e desvios padrão da resistência de união à dentina (MPa) imediata e após 6 meses de armazenamento em água dos sistemas adesivos convencionais

\begin{tabular}{|c|c|c|c|c|c|}
\hline \multirow{2}{*}{$\begin{array}{c}\text { SISTEMAS CONVENCIONAIS } \\
\text { ADESIVO (FABRICANTE) }\end{array}$} & \multicolumn{5}{|c|}{ VALORES DE RESISTÊNCIA DE UNIÃO (MPa) } \\
\hline & $\begin{array}{r}\left(\mathrm{N}^{\circ} \mathrm{de}\right. \\
\text { passos) }\end{array}$ & Solvente & Imediata & 6 meses & Referências \\
\hline Adper Scotchbond 1 & 2 & Água e etanol & $42,6 \pm 6,2$ & $38,4 \pm 6,9$ & Erhardt et al. 2008 \\
\hline One Step (Bisco) & 2 & Acetona & $27,2 \pm 4,9$ & $19,9 \pm 7,4$ & Reis et al. 2004 \\
\hline Prime \& Bond NT (Dentsply/Caulk) & 2 & Acetona e etanol & $38,4 \pm 5,6$ & $35,1 \pm 8,7$ & Osorio et al. ${ }^{1} 2008$ \\
\hline Scotchbond Multi-Purpose (3M ESPE) & 3 & Água & $42,6 \pm 5,5$ & $45,2 \pm 4,3$ & Reis et al. 2005 \\
\hline Single Bond (3M ESPE) & 2 & Etanol e água & $\begin{array}{l}26,7 \pm 4,9 \\
41,5 \pm 2,6 \\
41,0 \pm 6,9\end{array}$ & $\begin{array}{l}19,9 \pm 7,4 \\
28,0 \pm 2,6 \\
42,8 \pm 9,2\end{array}$ & $\begin{array}{l}\text { Reis et al. } 2004 \\
\text { Reis et al. } 2005 \\
\text { Osorio et al. } 2008\end{array}$ \\
\hline Syntac Single Component (Vivadent) & 3 & Água e acetona & $20,3 \pm 6,3$ & $20,4 \pm 7,5$ & Reis et al. 2004 \\
\hline
\end{tabular}

Tabela 2 - Médias e desvios padrão da resistência de união à dentina (MPa) imediata e após 6 meses de armazenamento em água dos sistemas adesivos autocondicionantes

\begin{tabular}{|c|c|c|c|c|c|}
\hline \multirow{2}{*}{$\begin{array}{l}\text { SISTEMAS AUTOCONDICIONANTES } \\
\text { ADESIVO (FABRICANTE) }\end{array}$} & \multicolumn{5}{|c|}{ VALORES DE RESISTÊNCIA DE UNIÃO (MPa) } \\
\hline & $\begin{array}{l}\left(\mathrm{N}^{\circ} \text { de }\right. \\
\text { passos) }\end{array}$ & Solvente & Imediata & 6 meses & Referências \\
\hline AdheSE (Ivoclar/Vivadent) & 2 & Água & $28,2 \pm 6,3$ & $27,2 \pm 7,7$ & Erhardt et al. 2008 \\
\hline Adper Prompt L-Pop (3M ESPE) & 1 & Água & $\begin{array}{r}24,3 \pm 3,1 \\
18,4 \pm 9,1 \\
\end{array}$ & $\begin{array}{r}16,9 \pm 4,1 \\
14,1 \pm 5,9 \\
\end{array}$ & $\begin{array}{l}\text { Reis et al. 2008; } \\
\text { Osorio et al. } 2008\end{array}$ \\
\hline AQBond (Sun Medical Co-EUA) & 1 & Água e acetona & $29,7 \pm 8,4$ & $22,1 \pm 9,4$ & Osorio et al. 2008 \\
\hline Clearfil Protect Bond (Kuraray) & 2 & Água & $39,2 \pm 5,2$ & $39,5 \pm 5,0$ & Erhardt et al. 2008 \\
\hline Clearfil SE Bond (Kuraray Co.) & 2 & Água & $\begin{array}{l}40,2 \pm 8,0 \\
40,6 \pm 4,7\end{array}$ & $\begin{array}{c}39,2 \pm 10,7 \\
26,6 \pm 4,1\end{array}$ & $\begin{array}{l}\text { Osorio et al. } 2008 \\
\text { Reis et al. } 2005\end{array}$ \\
\hline Etch \& Prime 3.0 (Degussa) & 1 & Água e etanol & $17,5 \pm 6,4$ & $14,0 \pm 4,8$ & Osorio et al. 2008 \\
\hline Futurabond & 1 & Acetona & $15,0 \pm 10,3$ & $11,1 \pm 3,9$ & Osorio et al. 2008 \\
\hline iBond (Heraeus Kulzer) & 1 & Água e acetona & $19,1 \pm 2,4$ & $18,1 \pm 4,8$ & Reis et al 2008 \\
\hline One-Up Bond (Tokuyama Co.) & 1 & Água & $68,4 \pm 8,7$ & $19.3 \pm 14,6$ & Hashimoto et al. 2003 \\
\hline $\begin{array}{l}\text { Optibond Solo self-etch Primer + Optibond Solo } \\
\text { Plus (Kerr) }\end{array}$ & 2 & Etanol & $36,2 \pm 4,0$ & $24,6 \pm 2,4$ & Reis et al 2005 \\
\hline Resulcin Aqua Prime & 2 & Solução aquosa & $15,0 \pm 6,2$ & $14,0 \pm 6,1$ & Osorio et al. 2008 \\
\hline Solist & 1 & Acetona & $31,2 \pm 5,5$ & $24,8 \pm 5,2$ & Osorio et al. 2008 \\
\hline Tyrian SPE + One Step Plus (Bisco) & 2 & Etanol & $24,1 \pm 3,7$ & $18,6 \pm 2,8$ & Reis et al. 2005 \\
\hline Xeno III (Dentsply De Trey) & 1 & Água e etanol & $30,0 \pm 1,2$ & $25,2 \pm 3,9$ & Reis et al. 2008 \\
\hline
\end{tabular}




\section{Discussão}

Diversos estudos têm sido realizados para avaliar a resistência de união imediata e após um período de armazenamento em água. Isso porque os sistemas adesivos são compostos por monômeros cada vez mais hidrófilos para que ocorra compatibilidade com o substrato dentinário que é inerentemente úmido (CASTRO, 2004; MALACARNE, 2005), contudo, estes materiais se tornaram mais suscetíveis à degradação ao longo do tempo por hidrólise (DE MUNCK, 2003 e 2005).

A água incorporada durante o procedimento de união pode ser da umidade intrínseca da dentina (PEREIRA, 1999) da técnica úmida e, da composição do adesivo, especialmente, o solvente (REIS, 2004). Os adesivos com monômeros hidrófilos são compatíveis com a umidade natural do substrato dentinário (MALACARNE, 2005), mas, o grau de umidade do substrato é fundamental no desempenho dos adesivos (REIS, 2003; PASHLEY, 2004) pois podem afetar significativamente a resistência de união (PEREIRA, 1999). De acordo com os dados observados nas tabelas $1 \mathrm{e} 2$, tanto os sistemas convencionais como os autocondicionantes mostraram maior redução da resistência de união após 6 meses de armazenamento em água quando o solvente era a acetona. Os sistemas adesivos contendo acetona mostraram altos valores de resistência de união imediata, porém tais valores após os 6 meses de armazenamento em água, de modo geral, reduziram significativamente (Tabelas 1 e 2).

Os sistemas adesivos hidrófilos absorvem mais água e perdem mais substância para o meio sendo que, a maior absorção ocorre nas primeiras 24 horas de armazenamento, independente do caráter hidrófilo do adesivo. A resistência de união também diminui nas primeiras 24 horas de armazenamento em água. O caráter mais hidrófilo dos adesivos torna as interfaces adesivas mais suscetíveis à permeação por água e, consequentemente, à degradação por hidrólise. Embora, a retenção imediata das resinas aos tecidos dentários necessite de tais características hidrófilas, em longo prazo, tal característica pode representar a autodestruição dessas resinas e, conseqüentemente, determinar o comprometimento da interface de união que constituem(Malacarne,2005).
A resistência de união de diferentes sistemas adesivos não são equivalentes quando armazenados a longo prazo conforme verificado nas tabelas $1 \mathrm{e} 2$, e um aumento da degradação da interface adesiva ao longo do tempo pode ser observada (DE MUNCK, 2003, 2005; SANO, 1999). Nos sistemas adesivos convencionais, zonas infiltradas incompletamente ao longo da camada híbrida, ou seja, fibras colágenas desprotegidas são mais suscetíveis à degradação em meio bucal (PASCHLEY, 2004).

A união dentina-resina formada por adesivos convencionais é propensa à degradação pela água, e os sistemas de 2 passos são mais suscetíveis do que os adesivos convencionais de 3 passos (DE MUNCK, 2003), corroborando com a revisão realizada (Tabela 1) onde pode ser observado que, exceto o Scotchbond Multi-Purpose (3M ESPE) que é um sistema convencional de 3 passos, todos os sistemas adesivos convencionais apresentaram diminuição dos valores da resistência de união após 6 meses de armazenamento em água.

Assim como o sistema convencional, Scotchbond Multi-Purpose (3M ESPE), o sistema autocondicionante Clearfil Protect Bond (Kuraray) não apresentou degradação após 6 meses de armazenamento em água (Tabelas 1 e 2). Entretanto, como os sistemas adesivos autocondicionantes geralmente são hidrofílicos, pela alta concentração de monômeros ácidos (KNOBLOCH, 2007), e, suscetíveis a sorção de água (TAY, 2003) o que pode influenciar a estabilidade ao longo do tempo. Os estudos "in vitro" mostraram que após um curto período de armazenamento, 6 meses, mostraram significativa redução na resistência de união (Tabela 2).

\section{Conclusão}

Pode ser observado que ao longo do tempo existe uma diminuição da resistência de união, mas não é uniforme para todos os sistemas adesivos, sendo dependente do adesivo aplicado e da sua composição (OSÓRIO, 2008; ARMSTRONG, 2003; YAMAUTI, 2003). 


\section{REFERÊNCIAS}

AMARAL, F. L. B.; COLUCCI, V.; PALMA-DIBB, R. G., CORONA, S. A. Assessment of in vitro methods used to promote adhesive interface degradation: a critical review. J Esthet Restor Dent., v.19, n.6, p.340-53, 2007.

ARMSTRONG, S. R.; VARGAS, M. A.; FANG, Q.; LAFFOON, J. E. Microtensile bond strength of a total-etch 3-step, total-etch 2-step, self-etch 2-step, and a self-etch 1-step dentin bonding system through 15 -month water storage. J Adhes Dent., v.5, n.1, p.47-56, 2003.

BOUILLAGUET, S.; GYSI, P.; WATAHA, J.C.; CIUCCHI, B.; CATTANI, M.; GODIN, C. Bond strength of composite to dentin using conventional, one-step, and self-etching adhesive systems. J Dent., v.29, n.1, p.55-61, 2001.

CARVALHO, R. M.; CARRILHO, M. R. O.; PEREIRA, L. C. G.; GARCIA, F. C. P.; MARQUEZINI JR., L.; SILVA, S. M. A., et al. Sistemas adesivos: fundamentos para aplicação clínica. Revista Biodonto; v.2, n.1, p.1-89, 2004.

CARRILHO, M. R.; CARVALHO, R. M.; TAY, F. R.; PASHLEY, D. H. Effects of storage media on mechanical properties of adhesive systems. Am J Dent., v.17, n.2, p.104-8, 2004.

CASTRO, A. K. B. B.; AMARAL, C. M.; AMBROSANO, G. M. B.; PIMENTA, L. A. F. Effect of sodium hypochorite gel on shear bond strength of one-bottle adhesive systems. Bras J Oral Sci, v.3, n.9, p.465, 2004.

DE MUNCK, J.; VAN MEERBEEK, B.; YOSHIDA, Y.; INOUE, S.; VARGAS, M.; SUZUKI, K. et al. Four-year water degradation of total-etch adhesives bonded to dentin. J Dent Res; v.82, n.2, p.136-40, 2003.

DE MUNCK, J.; VAN LANDUYT, K.; PEUMANS, M.; POITEVIN, A.; LAMBRECHTS, P.; BRAEM, M.; et al. A critical review of the durability of adhesion to tooth tissue: methods and results. J Dent Res. v.84, n.2, p.118-32, 2005.

ERNEST, C. P.; HOLZMEIER, M.; WILLERSHAUSEN, B. In vitro shear bond strength of self-etching adhesives in comparison to 4 th and 5th generation adhesives. J Adhes Dent.,v.6, n.4, p.293-9, 2004.

ERHARDT, M. C.; TOLEDANO, M.; OSORIO, R.; PIMENTA, L. A. Histomorphologic characterization and bond strength evaluation of caries-affected dentin/resin interfaces: effects of long-term water exposure. Dent Mater, v.24, v.6, p.786-98, 2008.

GARCIA, F. C. P.; D’ALPINO, P. H. P.; TERADA, R. S. S.; CARVALHO, R. M. Testes mecânicos para a avaliação laboratorial da união resina/dentina. Rev Fac Odontol Bauru, v.10, n.3, p.118-27, 2002.

HASHIMOTO, M.; OHNO, H.; SANO, H.; KAGA, M.; OGUCHI, H. Degradation patterns of different adhesives and bonding procedures. J Biomed Mater Res B Appl Biomater, v.66, n.1, p.324-30, 2003.

KNOBLOCH, L. A.; GAILEY, D.; AZER, S.; JOHNSTON, W. M.; CLELLAND, N.; KERBY, R. E. Bond strengths of one- and two-step self-etch adhesive systems. J Prosthet Dent, v.97, n.4, p.216-22, 2007.

LOGUERCIO, A. D.; UCEDA-GOMEZ, N.; CARRILHO, M. R.; REIS, A. Influence of specimen size and regional variation on long-term resin-dentin bond strength. Dent Mater, v.21, n.3, p.224-31, 2005.

MALACARNE J. Avaliação do comportamento de adesivos hidrófilos após armazenamento em água. Dissertação (Mestrado em Odontologia) - Faculdade de Odontologia de Piracicaba, da Universidade Estadual de Campinas, 2005.

MARTINS, G. C.; FRANCO, A. P. G.; GODOY, E. P.; MALUF, D. R.; GOMES, J. C.; GOMES, O. M. M. Adesivos dentinários. RGO, v.56, n.4, p.429-36, 2008.

NIKAIDO, T.; KUNZELMANN, K. H.; CHEN, H.; OGATA, M.; HARADA, N.; YAMAGUCHI, S., et al. Evaluation of thermal cycling and mechanical loading on bond strength of a self-etching primer system to dentin. Dent Mater, v.18, n.3, p.269-75, 2002.

OSORIO, R.; PISANI-PROENCA, J.; ERHARDT, M. C.; OSORIO, E.; AGUILERA, F. S.; TAY, F. R., et al. Resistance of ten contemporary adhesives to resin-dentine bond degradation. J Dent. v.36, n.2, p.163-9, 2008.

PASHLEY, D. H., TAY, F. R., YIU, C., HASHIMOTO, M.; BRESCHI, L.; CARVALHO, R.; et al. Collagen degradation by host-derived enzymes during aging. J Dent Res, v.83, n.3, p.216-21, 2004.

PEREIRA, P. N.; OKUDA, M.; SANO, H.; YOSHIKAWA, T.; BURROW, M. F.; TAGAMI, J. Effect of intrinsic wetness and regional difference on dentin bond strength. Dent Mater, v.15, n.1, p.46-53, 1999.

REIS, A.; LOGUERCIO, A. D.; AZEVEDO, C. L.; CARVAlHO, R. M.; SINGER, J. M.; GRANDE, R. H. Moisture spectrum of demineralized dentin for adhesive systems with different solvent bases. J Adhes Dent; v.5, n.3, p.183-92, 2003.

; LOGUERCiO, A. D.; CARVAlho, R. M.; GRANDE, R. H. Durability of resin dentin interfaces: effects of surface moisture and adhesive solvent component. Dent Mater, v.20, n.7, p.669-76, 2004.

; GRANDI, V.; CARLOTTO, L.; BORTOLI, G.; PATZLAFF, R.; RODRIGUES ACCORINTE, MDE L.; et al. Effect of smear layer thickness and acidity of self-etching solutions on early and long-term bond strength to dentin. $\mathbf{J}$ Dent, v.33, n.7, p.549-59, 2005.

;ALBUQUERQUE, M.; PEGORARO, M.; MATTEI, G.; BAUER, J. R.; GRANDE, R. H.; et al. Can the durability 
of one-step self-etch adhesives be improved by double application or by an extra layer of hydrophobic resin? J Dent; v.36, n.5, p.309-15, 2008.

SABOIA, V. P.; NATO, F.; MAZZONI, A.; ORSINI, G.; PUTIGNANO, A.; GIANNINI, M.; et al. Adhesion of a twostep etch-and-rinse adhesive on collagen-depleted dentin. J Adhes Dent., v.10, n.6, p.419-22, 2008.

SANO, H.; YOSHIKAWA, T.; PEREIRA, P.N.; KANEMURA, N.; MORIGAMI, M.; TAGAMI, J., et al. Long-term durability of dentin bonds made with a self-etching primer, in vivo. $\mathbf{J}$ Dent Res, v.78, n.4, p.906-11, 1999.

.; SHONO, T.; SONODA, H.; TAKATSU, T.; CIUCCHI, B.; CARVALHO, R. et al. Relationship between surface area for adhesion and tensile bond strength--evaluation of a micro-tensile bond test. Dent Mater, v.10, n.4, p.23640, 1994.

SCHREINER, R. F.; CHAPPELL, R. P.; GLAROS, A. G.; EICK, J. D. Microtensile testing of dentin adhesives. Dent Mater, v.14, n.3, p.194-201,1998.

TAY, F. R.; HASHIMOTO, M.; PASHLEY, D. H.; PETERS, M. C.; LAI, S. C.; YIU, C. K., et al. Aging affects two modes of nanoleakage expression in bonded dentin. J Dent Res, v.82, n.7, p.537-41, 2003. VAN LANDUYT, K. L.; KANUMILLI, P.; DE MUNCK, J.; PEUMANS, M.; LAMBRECHTS, P.; VAN MEERBEEK, B. Bond strength of a mild self-etch adhesive with and without prior acid-etching. J Dent; v.34, n.1, p.77-85, 2006.

VAN MEERBEEK, B.; DE MUNCK, J.; YOSHIDA, Y.; INOUE, S.; VARGAS, M.; VIJAY, P., et al. Buonocore memorial lecture. Adhesion to enamel and dentin: current status and future challenges. Oper Dent. v.28, n.3, p.215-35, 2003.

YAMAUTI, M.; HASHIMOTO, M.; SANO, H.; OHNO, H.; CARVALHO, R. M.; KAGA M. et al. Degradation of resindentin bonds using $\mathrm{NaOCl}$ storage. Dent Mater., v.19, n.5; p.399-405, 2003. 